\title{
Smart Business Process Management over Social Networks: Mechanism and Implementation
}

\author{
Yuechang Liu ${ }^{1,2}$, Yong Tang ${ }^{1}$, Muchao $\mathrm{Ke}^{2}$ and Yishan Fang ${ }^{2}$ \\ ${ }^{1}$ School of Computer Science, South China Normal University, Guangzhou China \\ ${ }^{2}$ School of Computer, Jiaying University, Meizhou China \\ ychangliu@gmail.com,ytang@scnu.edu.cn,751776425@qq.com, \\ ysfang@jyu.edu.cn
}

\begin{abstract}
Business Process Management (BPM) has gained great success in business and industry. However, business processes and workflow has been greatly changed by the emergence of social network media in recent years. It is necessary to study a mechanism of the seamless integration of BPM tools with existing social network platforms. This paper aims at building a social BPM system at the minimum cost on multiple existing popular public social networks. An integrated social BPM mechanism is proposed and implemented through abstract social network definition, abstract definition of BPM with Event-Driven Process Chains (EPC) model and open platforms of the social networks. The mechanism has the merits of stand-alone design, seamless integration and crossing platforms. Under the support of the mechanism people achieve seamless collaboration on public social networks.
\end{abstract}

Keywords: Social Networks, Business Process Management (BPM);, Collaboration

\section{Introduction}

Social networks play more and more important in people's life. It is reported that people in average spend 4 hours in updating his/her social networks and 4 hours in conversations with friends in the networks based on a 40 hours work week [3]. Moreover, as a new kind of media social media plays important role in business promotion, advertising, public relation and customer relation management. Social network tools facilitate people connecting to the Internet and offer magnanimity business opportunity. However, the variety of the tools (e.g., Email, IM, facebook, twitter, Weibo, blog, etc.,) further fragment people's life time and impose additional burden of switching among them on people.

Process and workflow management is effective way to improve work efficiency [3]. It is valuable to deploy process management tools on social networks, social Business Process Management (Social BPM) framework is proposed [2]. By using social BPM tools people define, execute and monitor business processes under the support of highly configurable on-line relations and information access control policy. It is reported that $80 \%$ of the adults use 1 to 3 social network tools, $40 \%$ use 2 or more such tools at the same time [3]. The variety of the tools fragments people's life time and imposes an additional burden of switching among the tools on them. It is highly required to study a social BPM that functions across multiple social media.

The first step is the abstraction of the social network and business process models. In this paper a social network involves three parts: a directed graph consisting of users as vertexes and user-to-user relations as edges, message that flows along the path on the edges, and a general role based access control policy. Business processes are modeled as Event-Driven Process Chain (EPC)[1]. To integrate EPC with social network structure, a variant of EPC, integrated EPC (iEPC) [5] is adopted in the paper. Then, the structure of 
crossing social media social BPM (CSMS-BPM for short) is formalized. To further understand the model, a run-on example is utilized through the paper.

The paper is organized as follows: the target problem is described and analyzed in the first section; then, the formal definition of CSMS-BPM is given in Section 2, followed by the section of architecture design. The last section is devoted to the conclusions, related and future works.

\section{Problem Description}

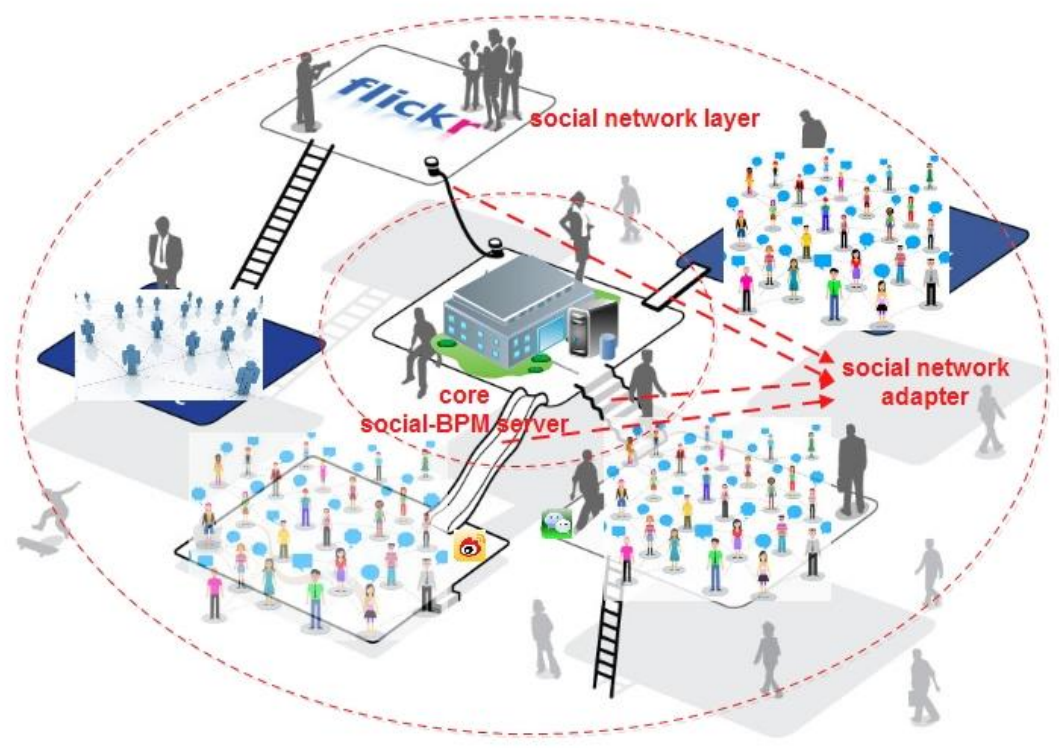

Figure 1. Social BPM Illustration

Consider the following scenario.

The BPM Company starts a new recruitment of the year. Difference from the past, the company decides to do the talents-hunter job on the Internet in this year. That is, the recruitment advertisement and application collection are all finished based on the social media. The managers of HR sector are responsible for the recruitment plan and requirement. All the other colleagues of HR sector are all responsible for posting the recruitment ads on social network platforms where they are registered. With all of the applications each HR receives and then are handed on to the HR manager, a screening procedure will be performed by the manager based on the applicant's resumes and the position requirements. The applications passing the first round screening will be replied with the offer of the opportunity of second-round interview. Otherwise they will be replied with a rejection letter. Then the process of the talents-huntering job is finished.

To summarize the task described in the above scenario, the process of the recruiting is managed by a core process engine. The information distribution and communication between the partitioners are accomplished by (not only one) social network tool(s).

\section{Basic Definitions and Notations}

Before the proposal of the mechanism, we first discuss the basic social network and integrated EPC definition.

In this paper, abstract social networks are viewed as consisting of relation networks of people (users), resources that can be manipulated and propagated through the relations (edges) of the network and the privilege for people to perform actions to it. 
The general social network definition is formalized as follows.

Figure $1^{1}$ pictorially illustrates the application context of system. To fulfill the requirements, a central social-BPM server engine is the core of the model, which serves as the coordinator, monitor for the processes and parser, producer of social media message for the mediator. People communicate with each other through either of the social network tools (typically flickr, facebook, Sina Weibo and WeChat are shown) which are registered on the BPM engine in the social network layer. Between the core social BPM server and the social networks, there are "bridges" that connect them. These "bridges" are adapters of the system which inherit common functions from the same interface.

Definition 1 (General Social Network, GSN) A general social network is composed of a directed graph (with the users as vertexes and users relations as the edges) and resources (each with a unique identity) that can be exchanged on the graph: $G S N=\left(G<V, E>, R E S, O P E R, R E S \_A C C\right)$, where $V=\left\{u_{1}, u_{2}, \ldots, u_{n}\right\}$

$\left.E=\left\{<u_{i}, u_{j}\right\rangle \mid \forall u_{i}, u_{j} \in V, u_{i} \neq u_{j}\right\}, R E S=\left\{r_{1}, r_{2}, \ldots\right\}, O P E R=\left\{o p_{1}, o p_{2}, \ldots, o p_{k}\right\}$, and

$R E S \_A C C \in V \times R E S \times O P E R \rightarrow\{$ true, false $\}$.

In Definition 1, RES is the set of resources that can be passed on the network, OPER the possible operations that can be performed to the resource and RES_ACC the function indicating the authorization of operations for specific user to perform on some resource.

The definition is adequate for most of existing public online social network (OSN) tools like facebook, twitter, Weibo, WeChat etc. The user relation graph is infrastructural backbone for all of OSN. The resource that flows on the graph is variable from tool to tool. For the instance of Sina Weibo, the resource can be short messages of 140 characters, web sites and "private message". For Tencent WeChat, the resource may be personal event, photos, web sites and instant text/voice message. The privilege of resource manipulation for users is better recognized as information access control policies, which is also differentiated for different OSN. Take Tencent WeChat for example, users can flexibly configure the resource access policy in the circle of friends.

Definition 2 (Integrated Event-Driven Process Chains, iEPC[5]). iEPC is defined to be a tuple $\mathrm{iEPC}=(\mathrm{E}, \mathrm{FN}, \mathrm{RN}, \mathrm{ON}, \mathrm{nm}, \mathrm{C}, \mathrm{A}, \mathrm{L})$, where $\mathrm{E}, \mathrm{FN}, \mathrm{RN}, \mathrm{ON}$ are respectively finite and non-empty set of events, function nodes, role nodes and object nodes for the process. The other components are defined as follows: 
$n m=n f \cup n r \cup n o$, where $: n f \in F N \rightarrow F$ assigns each function nodes to a function;

$n r \in R_{N} \rightarrow R$ assigns each role node to a role;

no $\in O_{N} \rightarrow R E S$ assigns each object to a resource.

$C=C_{C F} \cup C_{R} \cup C_{I N} \cup C_{\text {OUT }}$ is a finite set of logical connectors.

$-C_{C F}$ is the set of control-flow connectors;

$-C_{R}$ is the set of range connectors for role connectors;

$-C_{I N}$ the set of range connectors for input object nodes;

$-C_{\text {OUT }}$ is the set of range connectors for output object nodes.

$A=A_{C F} \cup A_{R} \cup A_{I N} \cup A_{\text {OUT }}$ is a set of arcs, where:

$-A_{C F} \subseteq\left(E \times F_{N}\right) \cup\left(F_{N} \times E\right) \cup\left(E \times C_{C F}\right) \cup\left(C_{C F} \times E\right) \cup\left(F_{N} \times C_{C F}\right) \cup\left(C_{C F} \times F_{N}\right)$ is the set of control-flow arcs;

$-A_{R} \subseteq\left(R_{N} \times F_{N}\right) \cup\left(R_{N} \times C_{R}\right) \cup\left(C_{R} \times F_{N}\right)$ the set of role arcs;

$-A_{I N} \subseteq\left(O_{N} \times F_{N}\right) \cup\left(O_{N} \times C_{I N}\right) \cup\left(C_{I N} \times F_{N}\right)$ the set of input arcs;

$-A_{\text {OUT }} \subseteq\left(F_{N} \times O_{N}\right) \cup\left(F_{N} \times C_{\text {OUT }}\right) \cup\left(C_{\text {OUT }} \times O_{N}\right)$ the set of outputarcs;

where $A_{R}, A_{I N}$ and $A_{\text {OUT }}$ are intransitive relations;

$L=l_{C}^{T} \cup l_{C}^{N} \cup l_{C}^{M} \cup l_{R}^{M} \cup l_{O}^{M} \cup l_{O}^{U}$ is the set of label assignments, where:

$-l_{C}^{T} \in R_{N} \rightarrow\{A N D, O R, X O R\}$ specifies the typeof control-flow connector,

$-l_{C}^{N} \in\left(C_{R} \cup C_{I N} \cup C_{\text {OUT }}\right) \rightarrow(\mathrm{N} \times(\mathrm{N} \times\{k\})) \cup\{(k, k)\}$ specifies lower bound and upperbound

of the range connector,

$-l_{C}^{M} \in\left(C_{R} \cup C_{I N} \cup C_{\text {OUT }}\right) \rightarrow\{M N D, O P T\}$ specifies if a role connector, an input connector or

an outputconnector is mandatory or optional,

$-l_{R}^{M} \in R_{N} \rightarrow\{M N D, O P T\}$ specifies if a role node is mandatory or optional,

$-l_{O}^{M} \in O_{N} \rightarrow\{M N D, O P T\}$ specifies if an object node is mandatory or optional,

$-l_{O}^{U} \in O_{N}^{I N} \rightarrow\{U S E, C N S\}$ specifies if an input object node is used or consumed,

where $O_{N}^{I N}=\operatorname{dom}\left(A_{I N}\right) \cap O_{N}$.

Under the Definition 2, Figure 2 graphically illustrates the iEPC model of the recruitment example.

Definition 3 (Crossing-Social Media Social BPM, CSMS-BPM). A CSMS-BPM is a tuple (U, SN, SBPM, $\downarrow_{S B P M}^{S N}, \uparrow_{S B P M}^{S N}$ ), where:

- $\mathrm{U}=\left\{\mathrm{u}_{1}, \mathrm{u}_{2}, \ldots, \mathrm{u}_{\mathrm{n}}\right.$, PESYS $\}$ the set of users that participate in the process (PESYS stands for the special user - process engine system),

- $\mathrm{SN}=\left\{\mathrm{sn}_{1}, \mathrm{sn}_{2}, \ldots, \mathrm{sn}_{\mathrm{m}}\right\}$ the set of social networks registered. Furthermore, we use $\mathrm{GRAH}\left(\mathrm{sn}_{\mathrm{i}}\right)$ to represent the directed graph implied by $\mathrm{sn}_{\mathrm{i}}$ and $\mathrm{ACP}\left(\mathrm{sn}_{\mathrm{i}}\right)$ for access control policy for $\mathrm{sn}_{\mathrm{i}}$.

- For each user $u_{j} \in U$, we use $s n_{i}\left(u_{j}\right)=\left\{\begin{array}{l}\text { not defined, if the } u_{j} \text { is not registered in } s n_{i} ; \\ v, \exists v \in G R A P H\left(s n_{i}\right) \text {, otherwise }\end{array}\right\}$

to refer to the corresponding user of $U$ in the $\mathrm{sn}_{\mathrm{i}}$. The process engine user, PESYS, is forced to be registered in each $\mathrm{sn}_{\mathrm{i}}$.

- $\mathrm{SBPM}=(\mathrm{E}, \mathrm{FN}, \mathrm{RN}, \mathrm{ON}, \mathrm{nm}, \mathrm{C}, \mathrm{A}, \mathrm{L})$ an integrated EPC where the role hierarchy implied in

is consistent with the $\mathrm{RH}$ on $\operatorname{nr}(\mathrm{RN})$. 


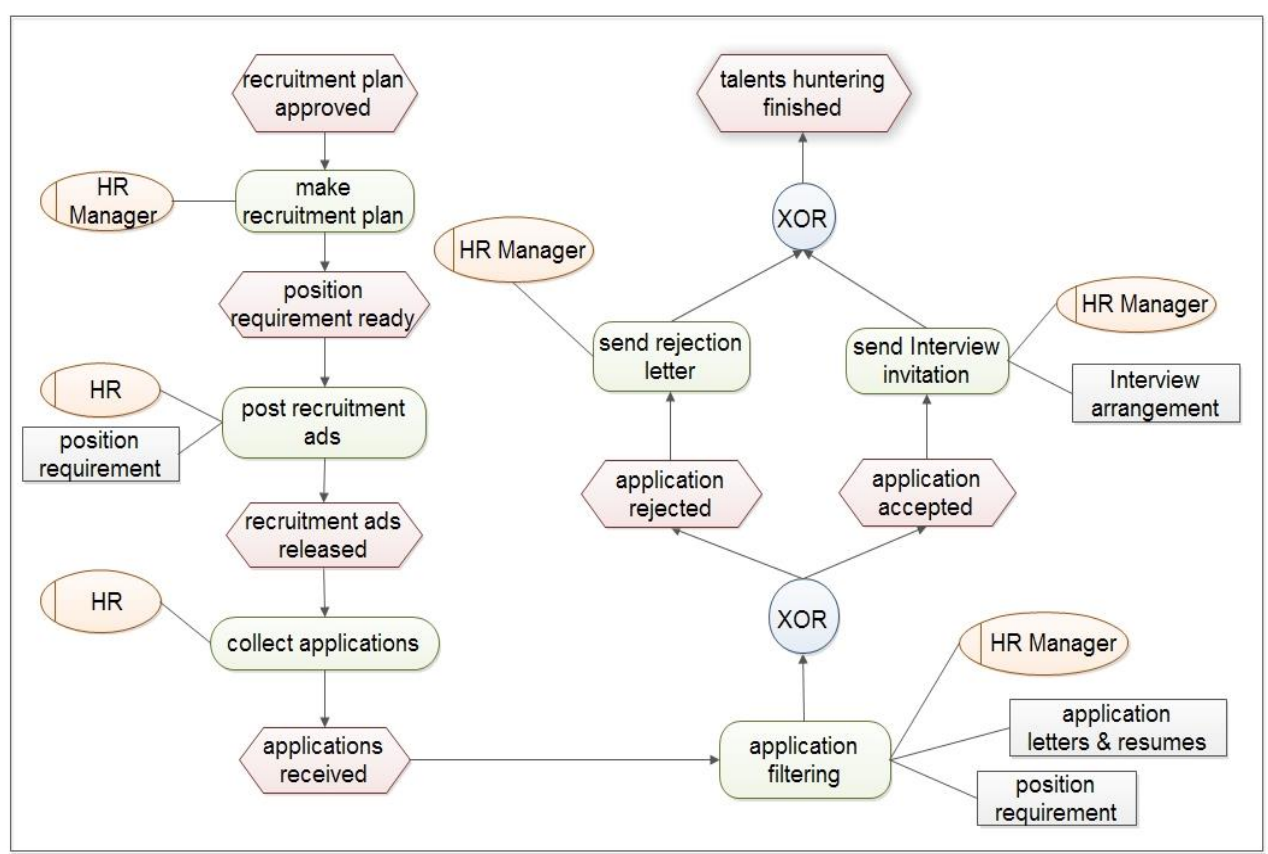

Figure 2. iEPC Model for the Recruitment Scenario

- $\quad \downarrow_{S B P M}^{S N}$ and $\uparrow_{S B P M}^{S N}$ are two operators that stand for the messaging with (the same) SN or between SBPM and SN (receiving and sending respectively). We use $\downarrow\left(s n_{i}, u_{x}, u_{y}, m\right)$ for the operation of $\operatorname{sn}_{i}\left(u_{y}\right)$, receiving message $m$ from the user $s n_{i}\left(u_{x}\right)$ and $\uparrow\left(s n_{i}, u_{x}, u_{y}, m\right)$ for $s n_{i}\left(u_{y}\right)$ sending $m$ to $\left\{A C P\left(u_{x}, u_{y}\right) \mid \forall u_{x}, u_{y} \in U\right.$, and $\left.u_{x} \neq u_{y}\right\} \times\left\{A C P\left(u_{x}, u_{y}\right) \mid \forall u_{x}, u_{y} \in U\right.$, and $\left.u_{x} \neq u_{y}\right\}$ $s n_{i}\left(u_{x}\right)$.

Figure 3 illustrates the integration of iEPC model and social networks. On the left is the connected existing social networks (including Weibo, facebook, WeChat, Linkedin, flickr, etc.)(The original photo can be found at). In the central of the networks is the core CSMS-BPM engine, which is connected to the networks in different forms with respect to the specific network type. The depiction vividly describes the crossing-social network feature of the CSMS-BPM engine. That is, resources (or messages) can be accessed across users from different social networks. On the right is the iEPC model of the running example. The model is connected to social networks with roles ( assigned to all of the users) and objects (i.e. The resources in the social networks.) The connection between the social networks and iEPC diagram represents the users (with roles) who are authorized to access the respective resources.

\section{CSMS-BPM Architecture: Design and Implementation}

The architecture is illustrated in Figure 4. As the abstraction of social networks, the Social Media Interface module separates various social network tools from the core social BPM engine. Like traditional BPM engine, the core social BPM engine serves as the core components that stores, schedules, controls and monitors the process definition and execution. The social media interface is the abstraction of the supported social networks which receives, parses and sends messages to the networks, and transforms messages to process objects (and reverse) meanwhile. 


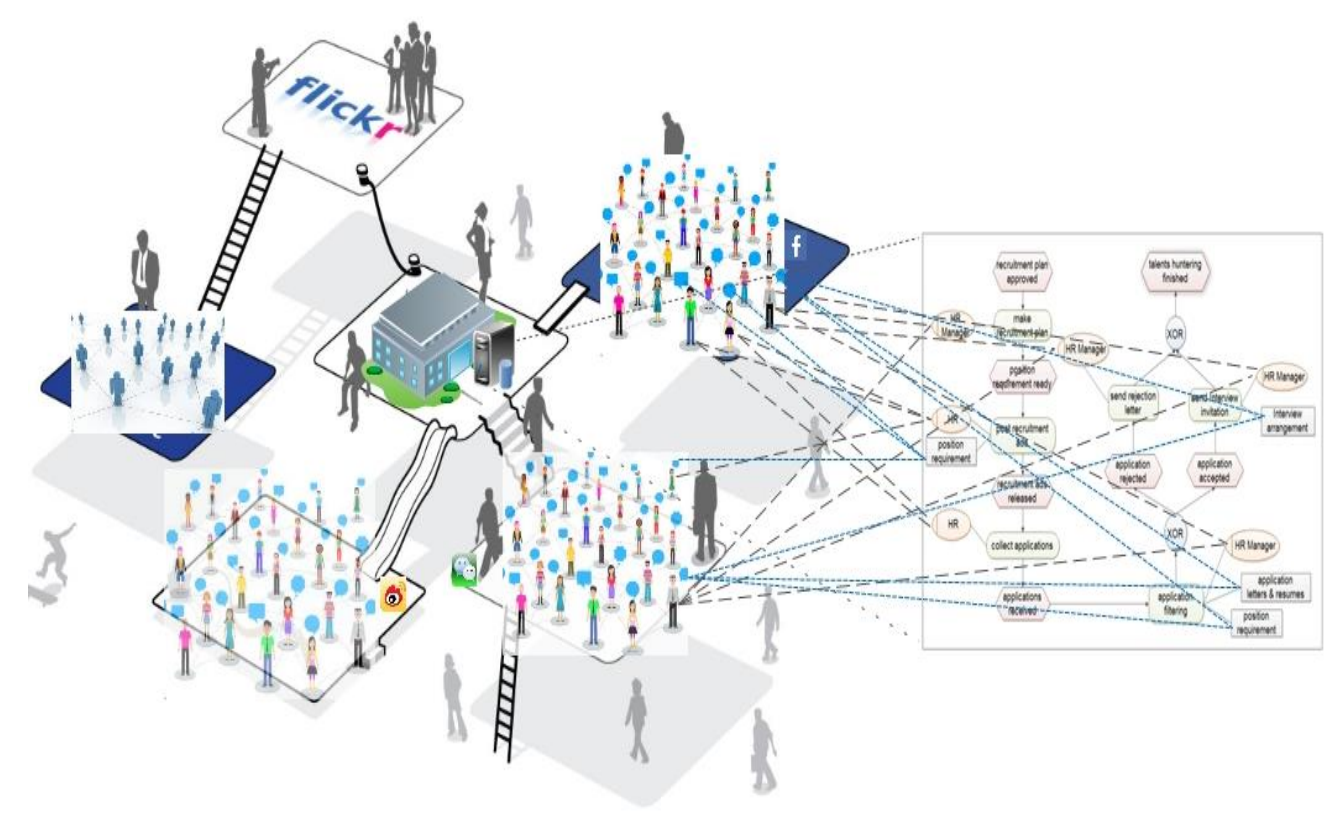

Figure 3. CSMS-BPM Illustrations

Same as normal BPM tools, CSMS-BPM runs by two steps: model definition and model execution. Model definition refers to the process model design, while model execution involves the practical execution of the process which the model defines.

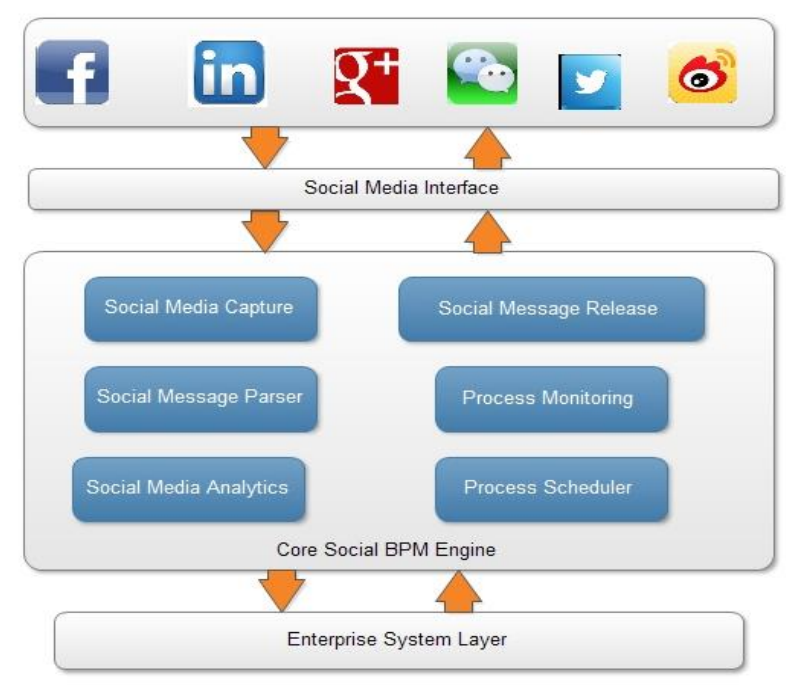

Figure 4. CSMS-BPM Architecture

\subsection{Process Design}

Figure 5 is the UML activity diagram which models the procedure that users create a process model in CSMS-BPM. The initiative users edit role and process definition as message text (in EPML format [2]) in some social network and send it to the PESYS user in the same social network. The core social BPM engine obtains the message of sn (PESYS) in some manner (e.g., Pull or push). The engine runs a message parser to construct the corresponding EPC model and store it. The model can be referred by a unique ID when someone activates it. 


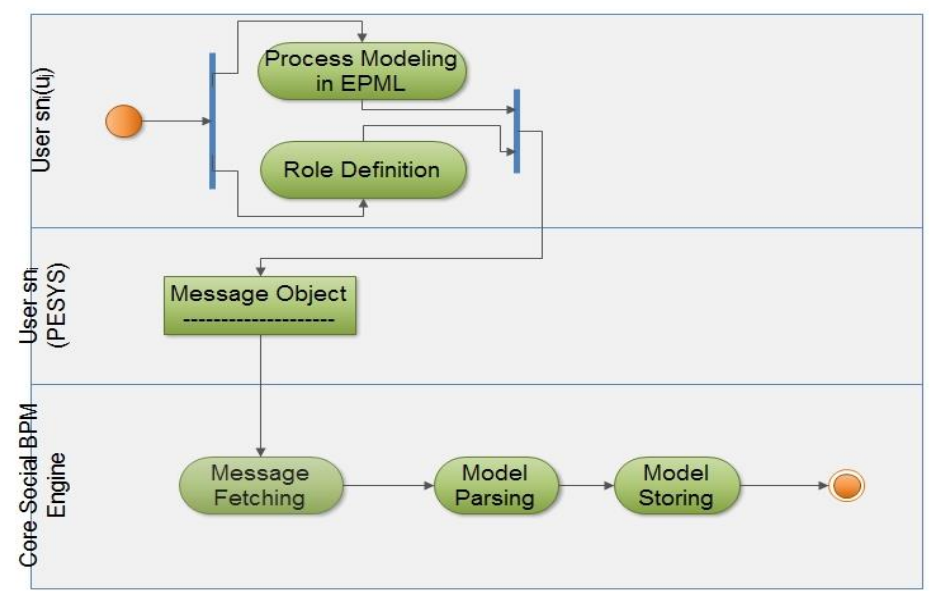

Figure 5. Model Definition

\subsection{Process Execution}

The model execution process is illustrated in Figure 6. The procedure starts with process initiation by some user through sending formatted message with some necessary parameters (e.g., Role, workflow model ID, resource, etc.,) to sni(PESYS) on some social network sni. Afterwards the procedure is controlled by the core BPM engine and the interaction between users and the user sni (PESYS).

During the execution of the process, users can whenever query the status of the process simply by sending some formatted message to sni (PESYS).

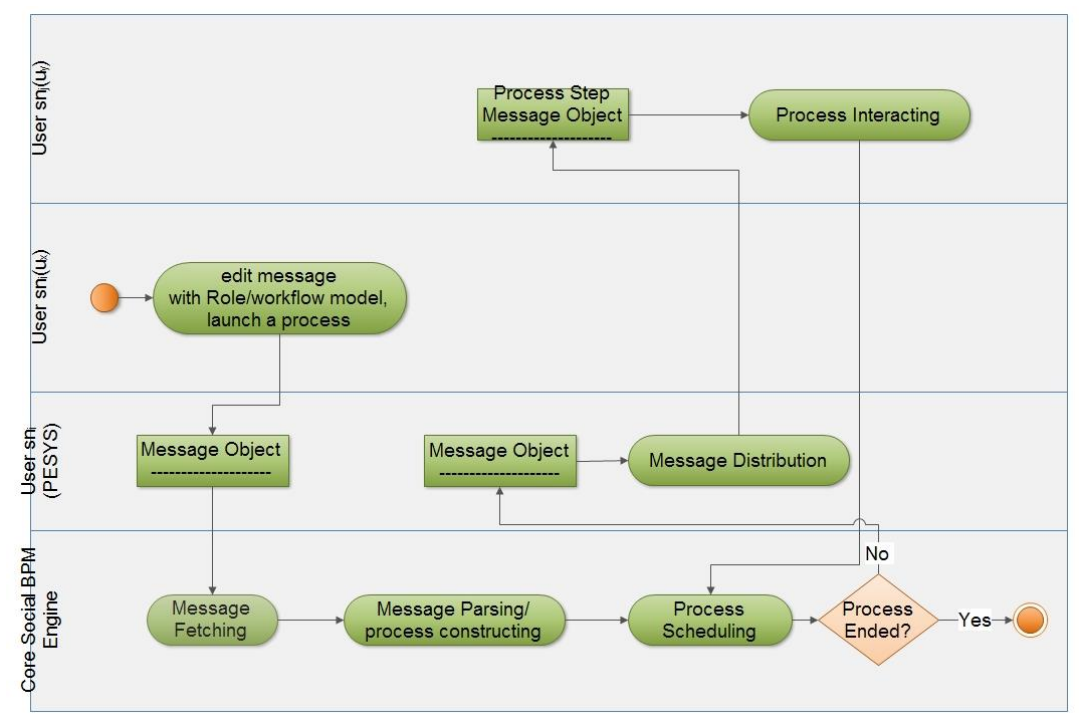

Figure 6. Process Execution

\subsection{Implementation}

The CSMS-BPM prototype system is under implementation. Currently, only OSN interface with Sina Weibo is adopted. The implementation is based on the Weibo Open Platform (http://open.weibo.com/). Once a Weibo user logged in and add PESYS as friend, he can design a process by sending process definition text in EPML format to PESYS. Figure 7 and Figure 8 are the basic GUI of the implemented prototype system, which illustrates the dashboard and workflow management page respectively. The dashboard is mainly used for social communication function (which is synchronized with 
public social networks in, e.g., Weibo for now, at the back end) and information summary representation, while the process and workflow page is centered with the functions like process creation, initiation, task operation and communication. social networks in that it extends EPC with role and resource nodes to gain configurability. CSMS-BPM model is formally defined in this paper and illustrated with a running example.

\section{Conclusions, Related and Future Work}

In this paper, an architecture called CSMS-BPM that integrates traditional BPM with social networks is proposed. CSMS-BPM models the social networks as essential directed graph and general RBAC access control policy. To adapt with the social network definition, iEPC [5] for process modeling is adopted. iEPC can be organically integrated with essential social networks in that it extends EPC with role and resource nodes to gain configurability. CSMS-BPM model is formally defined in this paper and illustrated with a running example.

The theory and application of social BPM has been intensively studied in recent years. It is generally recognized that social BPM is the integration of traditional BPM and social media. There are three families of solutions distinguished by the bias in the integration: tradition BPM biased, social tools biased, and emerging suites. Among of those families, social BPM suites are promising but still in their infancy. As a case of this family, IBM announced Blueworks Live [9] in mid-November 2010, but the actual social features are still under definition. Some technical community and funded research projects aim at extending traditional BPM methods towards social collaboration, like the Process Live tool from ARIS community [10]. Piero Fraternali, et al., proposed a model-driven framework for social BPM applications [11]. In their research, they designed a five-layer social BPM model, implemented in their WebRatio suites based on the socially extended BPMN 2.0 process modeling language and WebML (web modeling language). The research of this paper aims at designing a light-weighted social BPM mechanism, which uses existing typical social media and BPM platform. The mechanism is formalized with formal process modeling language EPML (the EPC markup language in XML) [2] and abstract social network definition. It differs from the relevant mechanism in the literature in the process modeling language and integration tightness with social media. CSMSBPM serves as the bridge between existing BPM tools and social media. In the next step, the language that is more suitable for the representation of processes in the social media will be deeply studied. Moreover, based on the formal representation language the system properties of CSMS-BPM will be further explored. In the future development, more effort will be dedicated to designing CSMS-BPM to be an adapter middle-ware of BPM and social tools.

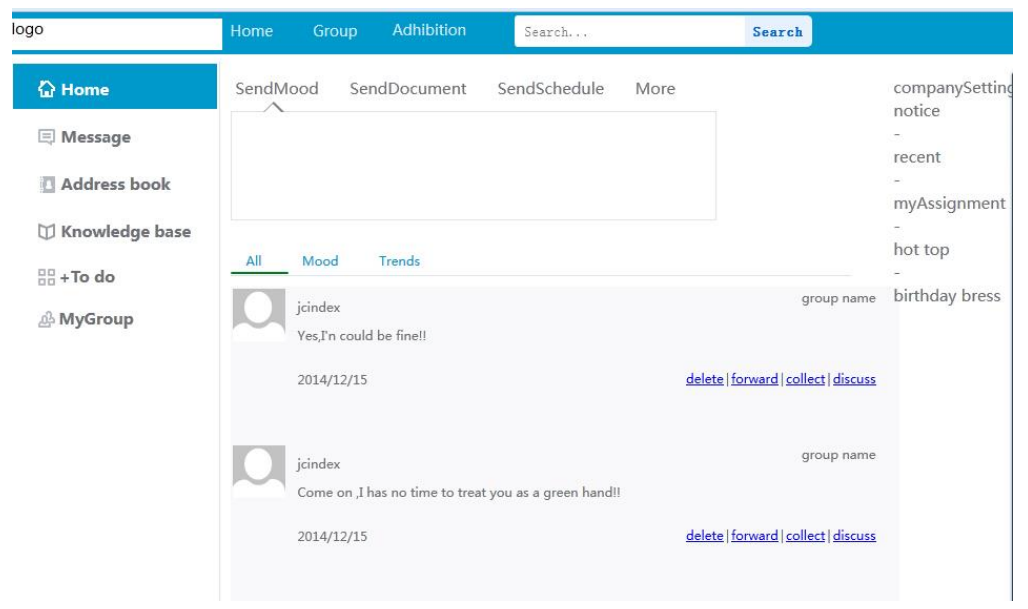

Figure 7. Dashboard Page 


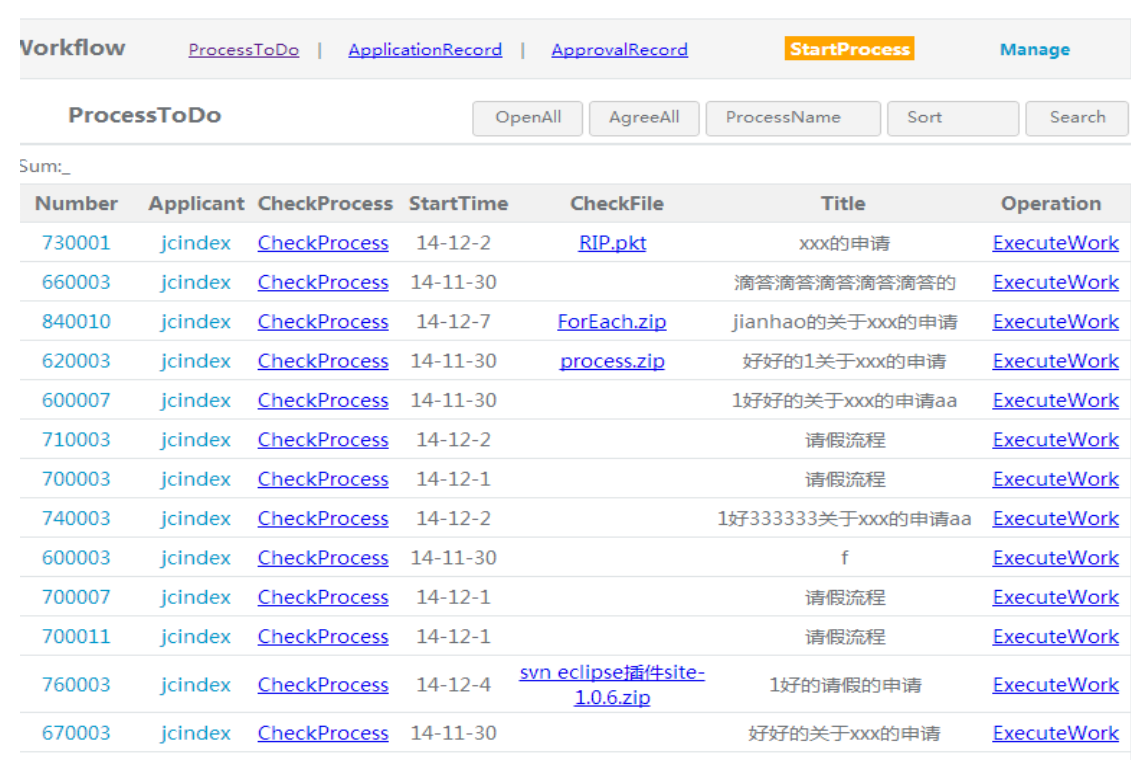

Figure 8. Process (Workflow) Management Page

\section{Acknowledgment}

The research is supported by following projects: the National Science Foundation (No. 60970044 and No. 61272067) in part and Jiaying University Natural Science Research Project "Research and Application of Collaboration Mechanism in Social Networks".

\section{References}

[1] A. W. Scheer, “ARIS - Business Process Frameworks", Springer, Berlin, 3rd edition, (1999).

[2] J. Mendling and M. Nüttgens, "EPC markup language (EPML): an XML-based interchange format for event-driven process chains (EPC)", ISeB, vol. 4, no. 3, (2006) July, pp. 245-263.

[3] S. Krishnamurthy, "Social Process Design, Execution and Intelligence for a better Customer Experience", Infosys, white paper, (2011).

[4] M. L. Rosa, M. Dumas, A. H. M. ter Hofstede, J. Mendling and F. Gottschalk, "Beyond Control-Flow: Extending Business Process Configuration to Roles and Objects", in Conceptual Modeling - ER 2008, Q. Li, S. Spaccapietra, E. Yu and A. Olivé, Springer Berlin Heidelberg, (2008), pp. 199-215.

[5] M. La Rosa, M. Dumas, A. H. M. ter Hofstede and J. Mendling, Configurable multi-perspective business process models, Information Systems, vol. 36, no. 2, (2011), pp. 313-340.

[6] M. Smiciklas, "Social Media Workflow", (2013) January, Via http://www.intersectionconsulting.com/2013/social-media-workflow/.

[7] W. Robison, "Social Workflow - A Future of Work Trend", Technology, (2013) January 27.

[8] D. Ferraiolo, J. Cugini and D. R. Kuhn, "Role-based access control (RBAC): Features and motivations", Proceedings of 11th annual computer security application conference, (1995), pp. 241-48.

[9] “[IBM11] IBM”, IBM BlueWorks Live, (2011) May, https://www.blueworkslive.com.

[10] "Process Live", http://processlive.com/.

[11] M. Brambilla, P. Fraternali and C. V. Ruiz, "A model-driven approach to Social BPM applications. In Social BPM Handbook", BPM and Workflow Handbook series, Future Strategies, USA, (2011) June, pp. 95-112.

[12] Z. Jun and W. Ji-Yi, "Provable Secure Efficient Arbitrated Quantum Signature Scheme", Journal of Beijing University of Posts and Telecommunications, vol. 36, no. 2, (2013), pp. 113-116. 


\section{Authors}

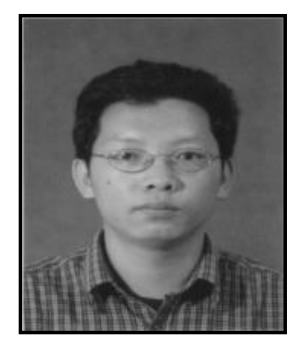

Yuechang Liu, $\mathrm{Ph} . \mathrm{D}$ in computer software and theory, postdoctoral researcher in School of Computer Science, South China Normal University. His research interest include knowledge engineering, social network analysis .

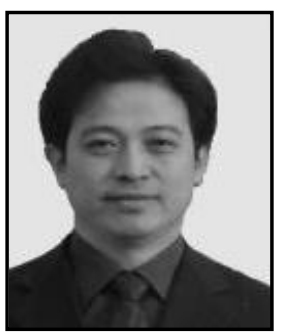

Yong Tang, Professor and Ph.D supervisor in computer science in School of Computer Science, South China Normal University. His research interests include temporal database, social computing and computer supported collaborative work. 